\title{
Case of the Very Rare Gallbladder Paraganglioma
}

\author{
Shukla Shreya ${ }^{1}$ Ritu Kashikar ${ }^{1}$ Sinhasan R. Shraddha ${ }^{1}$ Desai Shrinivas ${ }^{1}$ \\ ${ }^{1}$ Department of Radio-Diagnosis, Jaslok Hospital and Research \\ Centre, Mumbai, Maharashtra, India \\ Address for correspondence Shreya Shukla, Tata Memorial Hospital, \\ Dr. Ernest Borges Rd., Parel East, Parel, Mumbai - 400012 , \\ Maharashtra, India (e-mail: drshreyashukla@gmail.com).
}

Indian J Radiol Imaging 2021;31:693-696.

\author{
Abstract \\ Keywords \\ - CECT head and neck \\ tumors \\ - DOTATATE PET/CT \\ - gallbladder \\ paraganglioma \\ - glomus tumors \\ - multiple \\ paragangliomas
}

Gallbladder paraganglioma is a very rare condition, and only a few cases have been reported in the literature. Herein, we present and discuss the clinical and radiological findings of a 72-year-old woman who complained of heaviness, pain, and on and off watery discharge from the left ear for 1 year and reduced hearing in both ears. She was thoroughly investigated radiologically where multiple paragangliomas were found with the discovery of the very rare gallbladder paraganglioma. There is a need to realize the importance of thorough radiological evaluation in cases of head and neck paraganglioma to look for more such lesions in the body for early identification of patients with familial paraganglioma syndromes. With this practice, gene mutation carriers can be identified early in the course of their disease and brought to surgical attention before their disease becomes extensive and potentially life-threatening.

\section{Introduction}

Gallbladder paraganglioma is a very rare tumor and very few cases have been reported in the literature. Many of these cases are asymptomatic and reported to have been found incidentally during an operation performed for cholelithiasis, cholecystoduodenal fistula repair for recurrent hematemesis, and Roux-en-Y gastric bypass operation for morbid obesity. Paraganglia differentiate from neural crest cells during the embryonic period and make up a dispersed neuroendocrine system near or in the autonomic nervous system, with extension from the skull base to the pelvic floor. Paragangliomas are neoplasms originating from paraganglia. The most common location of paraganglioma is the adrenal medulla, which is defined as pheochromocytoma. The second most frequent region is the carotid body. Paragangliomas have been reported in the retroperitoneum, head and neck, paranasal sinuses, urinary bladder, and many different organs. Paragangliomas are very rare in the gallbladder. Herein, we present a case of gallbladder paraganglioma, a rarity, discovered on thorough radiological evaluation with whole body cross-sectional imaging. This case report aims to review the literature on this rare condition and stress on the need for a thorough evaluation of cases of paraganglioma of the head and neck to look for more lesions in the body for identification of patients with familial paraganglioma syndromes. This shall facilitate the identification of gene mutation carriers early in the course of their disease so that they are brought to surgical attention before their disease becomes extensive and potentially lifethreatening.

\section{Case History}

A 72-year-old woman presented with complaints of heaviness, pain, and on and off watery discharge from the left ear since the last 1 year and reduced hearing in both ears, with the left ear being affected more than the right. On examination, a retracted eardrum was seen on the left side, which otherwise appeared healthy, with a normal-appearing external auditory canal. Highresolution computed tomography (HRCT) temporal bone was
DOI https://doi.org/ 10.1055/s-0041-1736162. ISSN 0971-3026. (c) 2021. Indian Radiological Association. All rights reserved.

This is an open access article published by Thieme under the terms of the Creative Commons Attribution-NonDerivative-NonCommercial-License, permitting copying and reproduction so long as the original work is given appropriate credit. Contents may not be used for commercial purposes, or adapted, remixed, transformed or built upon. (https://creativecommons.org/ licenses/by-nc-nd/4.0/)

Thieme Medical and Scientific Publishers Pvt. Ltd., A-12, 2nd Floor, Sector 2, Noida-201301 UP, India 


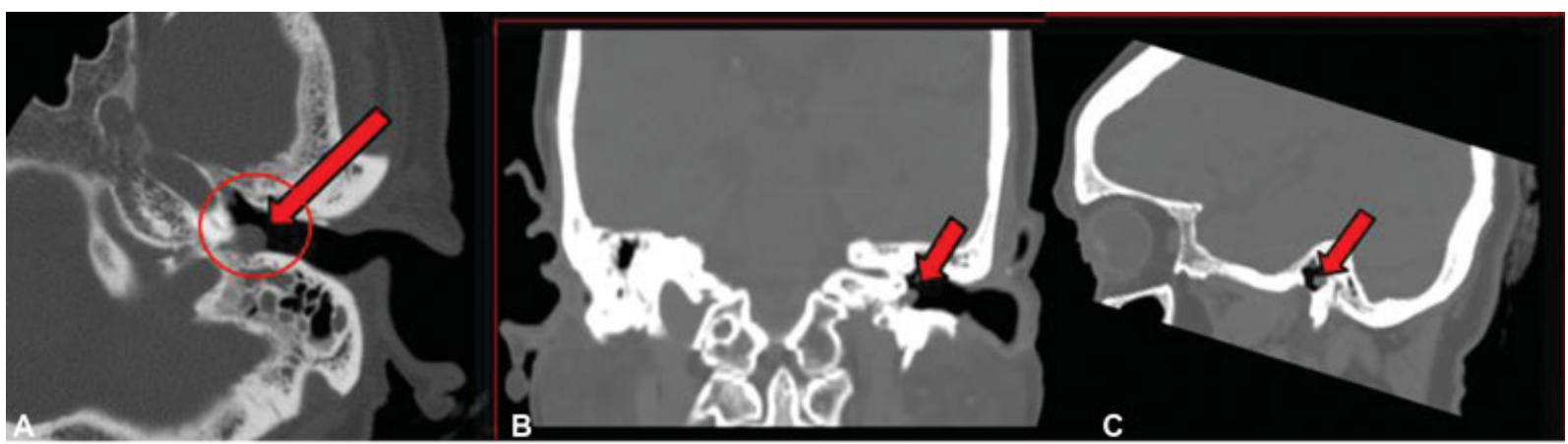

Fig. 1 Axial (A), Coronal (B), Sagittal (C) non-contrast CT scan of temporal bone showing soft tissue density lesion in the left mastoid antrum extending into the left hypotympanum. $\mathrm{CT}$, computed tomography.

done that showed a soft tissue density lesion in the left mastoid antrum extending into the hypotympanum ( - Fig. 1A-C). The external ear canal, facial nerve canal, cochlea, and semicircular canals were intact. Subtle erosions were seen involving the medial wall of the mastoid antrum and posterior wall of the middle ear. There was no evidence of ossicular destruction. The radiological suspicion of glomus tympanicum was raised. We suggested a contrast-enhanced computed tomography (CECT) of the head and neck to look for the multicentricity of glomus tumors. The contrast study revealed multiple soft tissue mass lesions showing intense homogenous enhancement in the left hypotympanum extending into jugular fossa and bilateral carotid spaces (-Fig. 2A-D).

A Ga-DOTATATE positron emission tomography-computed tomography (PET/CT) scan was done to screen from the skull base to the pelvic floor to look for more similar lesions. The CECT revealed multiple intensely enhancing lesions in

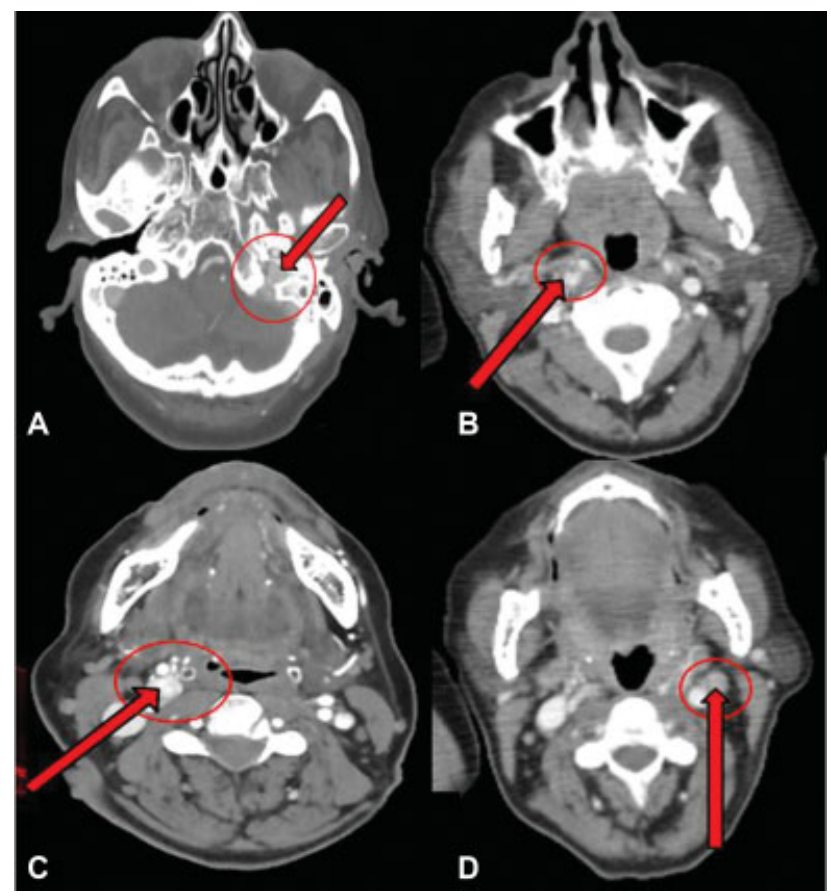

Fig. 2 Axial CECT scan of the head and neck revealing multiple intensely enhancing soft tissue lesions in the left hypotympanum extending into the jugular fossa (A) and bilateral carotid spaces (B-D). CECT, contrast-enhanced computed tomography.
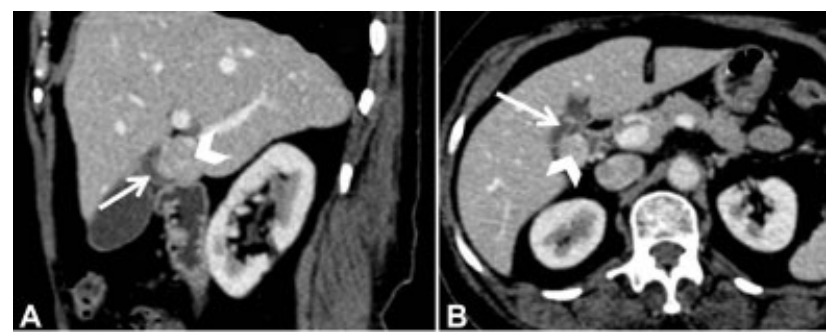

Fig. 3 Sagittal (A) and Axial (B) CECT images of the abdomen showing enhancing lesion (marked with arrowhead) concerning the neck (marked with an arrow) of the gall bladder. CECT, contrast-enhanced computed tomography.

the head and neck region ( - Fig. 2A-D). Another similar soft tissue lesion was seen in the neck of the gallbladder showing avid enhancement (-Fig. $\mathbf{3 A}$ and $\mathbf{3 B}$ ). This lesion measured $\sim 2.3 \times 2.2 \mathrm{~cm}$ in size. The rest of the gallbladder was unremarkable. The lesions in the head and neck showed strong somatostatin receptor (SSTR) expression $\left(\mathrm{SUV}_{\max } 89.5\right)$. The lesion in the gallbladder region also showed intense GaDOTATATE uptake $\left(\mathrm{SUV}_{\max }\right.$ 90.7) (-Fig. 4). A radiological diagnosis of multiple paragangliomas was made with the striking finding of a rare gallbladder paraganglioma. Histopathology report of the lesion in the left hypotympanum confirmed the lesion to be paraganglioma. On detailed inquiry, there was no history of a similar condition in any family member. The patient was given symptomatic treatment for her ear complaints. No major invasive procedure was done, given the age of the patient and an asymptomatic course thereafter.

\section{Discussion}

Paragangliomas are neoplasms originating from paraganglia. Paraganglia differentiate from neural crest cells during the embryonic period and make up a dispersed neuroendocrine system near or in the autonomic nervous system, with extension from the skull base to the pelvic floor. An exception is paraganglioma of the adrenal medulla, which is universally known as pheochromocytoma. Paragangliomas located outside the adrenal gland have been designated as extra-adrenal paragangliomas. ${ }^{1,2}$ 


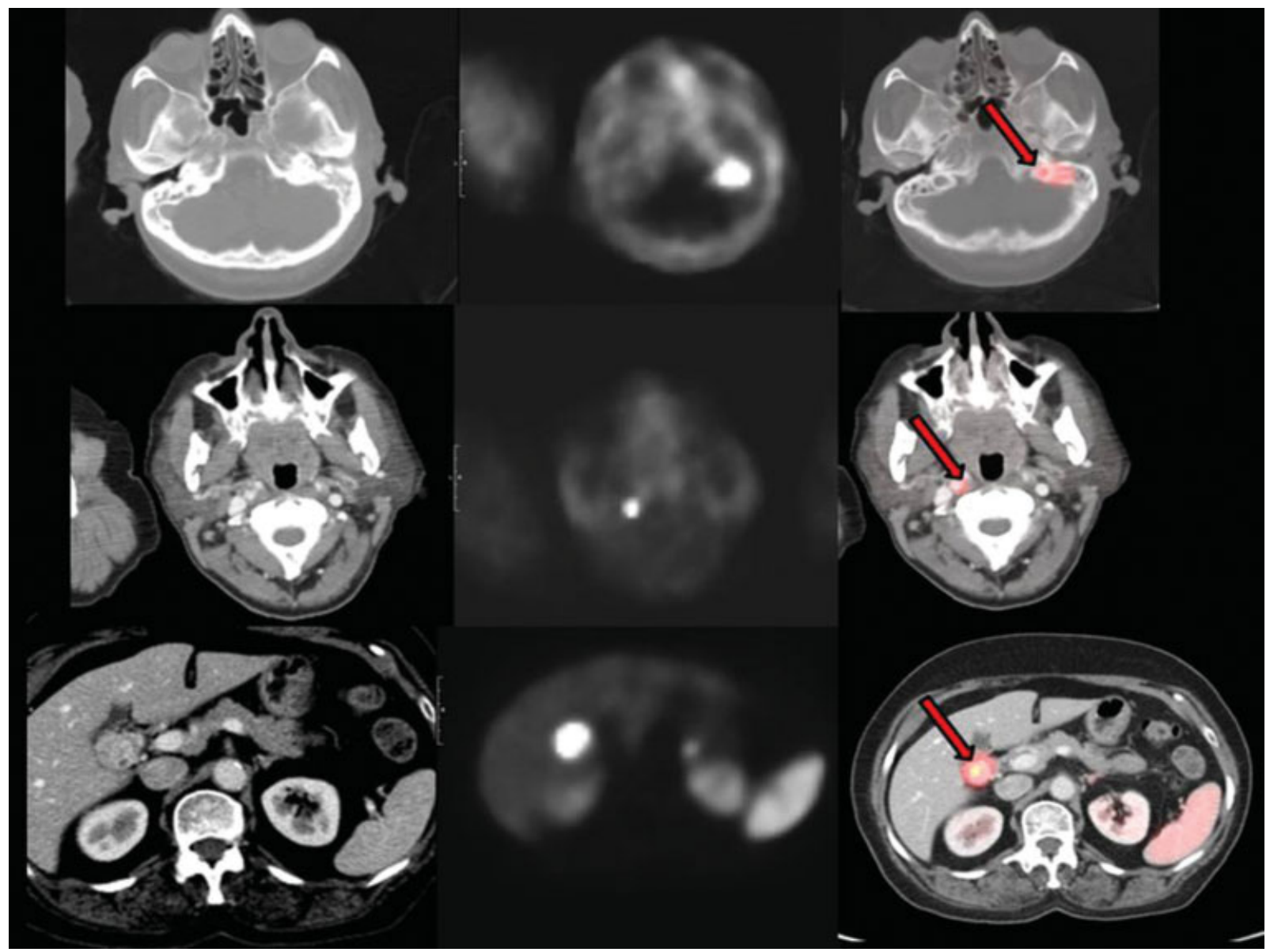

Fig. 4 Axial DOTATE PET-CT scan images showing multiple SSTR expressing intensely enhancing lesions in the head and neck (SUV $\left.\mathrm{max}_{89} 89.5\right)$. Another lesion is seen in the region of gall bladder showing avid enhancement and intense Ga-DOTATATE uptake (SUV $\max 90.7)$. PET-CT, positron emission tomography-computed tomography; SSTR, strong somatostatin receptor.

Paragangliomas of the head and neck are closely aligned with the distribution of the parasympathetic nervous system, and they most commonly arise at the carotid body, followed by lesions within the jugular foramen or middle ear and along the course of the vagus nerve. ${ }^{3}$ Besides, these tumors are found in other less common sites including the orbit, nasal cavity, nasopharynx, thyroid gland, larynx, pineal gland, and cheek. Extra-adrenal paragangliomas of the abdomen arise predominantly from paraganglia closely related to the sympathetic nervous system and are located in the retroperitoneum. Other less common locations of abdominal paragangliomas include the gallbladder, urinary bladder, prostate, spermatic cord, uterus, and duodenum.

There is limited literature regarding gallbladder paraganglioma, according to which most of the cases were discovered incidentally during cholecystectomy, and some of these presented with right upper quadrant pain. One case had manifested with gastrointestinal bleeding. ${ }^{4}$ In our case, the asymptomatic gallbladder mass was discovered incidentally on PET-CT images of the abdomen.

Biliary system paragangliomas are predominantly seen in females and are discovered in the fifth to sixth decades of life. Because these tumors are nonfunctioning, they are typically discovered secondary to their complications, such as obstructive jaundice, right upper quadrant pain, and gastroin- testinal bleeding, or incidentally during gallbladder or unrelated surgery. They are most commonly located in subserosa. Interestingly, all these tumors are nonfunctioning. Thus, they mimic chromaffin-negative extra-adrenal tumors found in the head, neck, and mediastinal region, as opposed to the extraadrenal retroperitoneal paragangliomas.

Extra-adrenal tumors are more commonly malignant than adrenal tumors. Distinguishing malignant tumors from benign paraganglioma is not histologically easy. None of the suggested histologic features can independently predict the biological behavior of tumors. However, coarse nodularity, absence of hyaline globules positive for periodic acid-Schiff, and an extra-adrenal location are known to be suggestive of a malignant nature. Distant metastasis, which can be seen on imaging, is the most reliable and definitive indicator of malignancy. ${ }^{5,6}$

Primary gallbladder paragangliomas theoretically arise from the primordia of hepatic plexus, which innervates the gallbladder, and are formed from sympathetic and parasympathetic fibers of the left vagus nerve and celiac plexus. ${ }^{7}$ It is important to realize that primary gallbladder paragangliomas, although rare, may occur, and should be considered in the differential diagnosis of gallbladder lesions. A careful search should be performed, as in this case, for the possible association with familial paraganglioma syndromes. 
Extra-adrenal paragangliomas can be found in practically every site in which paraganglia are known to occur. Although these tumors occur in a variety of anatomic locations, they have nearly identical imaging features, including a homogeneous or heterogeneous hyperenhancing soft-tissue mass at $\mathrm{CT}$, multiple areas of signal void interspersed with hyperintense foci (salt-and-pepper appearance) within the tumor mass at magnetic resonance imaging (MRI), and an intense tumor blush with enlarged feeding arteries at angiography. Therefore, when hypervascular masses are seen in specific locations of the body, the possibility of paragangliomas should always be considered.

Most paragangliomas are solitary and arise sporadically, they can be multicentric, and familial occurrence is wellrecognized in them. The incidence of multicentricity is $\sim 10 \%$ of the total cases. ${ }^{8}$ Familial paragangliomas constitute $~ 10 \%$ of cases, and 35 to $50 \%$ of cases of familial paragangliomas have multicentric tumors. ${ }^{9,10}$ Thus, careful family history is required, and relatives of a familial case should be screened for paraganglioma. These familial tumors are commonly associated with these conditions: multiple endocrine neoplasia (MEN IIA and IIB) and neuroectodermal syndromes (tuberous sclerosis, neurofibromatosis, and von Hippel-Lindau disease). Paragangliomas may also occur as part of Carney's triad. Familial paragangliomas are most commonly observed after the third decade of life although they can be seen in the early teen years. Another characteristic feature of familial tumors is multicentricity and bilaterality. So far, only one biliary system paraganglioma with a familial association has been reported. ${ }^{11}$ Our patient had no family history of endocrine neoplasia.

Molecular genetic screening of all head and neck paraganglioma patients for mutations of the genes $S D H B, S D H C$, and $S D H D$ is recommended. Whenever a mutation is detected, the affected patient should undergo a thorough clinical examination, an MRI with contrast medium of the head and neck, the thorax and the abdomen as well as catecholamines or metanephrines in the plasma or 24-hour urine.

The identification of patients with familial paraganglioma syndromes will, in addition with follow-up examinations, lead to early detection of head and neck paragangliomas and pheochromocytomas in the future. In turn, carriers identified early in the course of their disease can be brought to surgical attention before their disease becomes extensive and potentially life-threatening.

\section{Funding}

None.

Conflicts of Interest

None declared.

\section{References}

1 Lack EE. Paraganglioma. In: Sternberg SS, ed. Diagnostic Surgical Pathology. 2nd ed. New York, NY: Raven Press; 1994:599-621

2 Rosai J. Adrenal gland and other paraganglia. In: Rosai J, ed. Ackerman's Surgical Pathology. 8th ed. St. Louis: Mosby; 1996: 1015-1058

3 Duncan AW, Lack EE, Deck MF. Radiological evaluation of paragangliomas of the head and neck. Radiology 1979;132(01):99-105

4 Oztas M, Ersoz N, Ozerhan I, et al. Gallbladder paraganglioma: report of a rare case. Gülhane Tip Derg 2015;57:73-75

5 Pommier RF, Vetto JT, Billingsly K, Woltering EA, Brennan MF. Comparison of adrenal and extraadrenal pheochromocytomas. Surgery 1993;114(06):1160-1165, discussion 1165-1166

6 Linnoila RI, Keiser HR, Steinberg SM, Lack EE. Histopathology of benign versus malignant sympathoadrenal paragangliomas: clinicopathologic study of 120 cases including unusual histologic features. Hum Pathol 1990;21(11):1168-1180

7 Miller TA, Weber TR, Appelman HD. Paraganglioma of the gallbladder. Arch Surg 1972;105(04):637-639

8 Young AL, Baysal BE, Deb A, Young WF Jr. Familial malignant catecholamine-secreting paraganglioma with prolonged survival associated with mutation in the succinate dehydrogenase $B$ gene. J Clin Endocrinol Metab 2002;87(09):4101-4105

9 Magliulo G, Zardo F, Varacalli S, D'Amico R. Multiple paragangliomas of the head and neck. An Otorrinolaringol Ibero Am 2003;30 (01):31-38

10 Grufferman S, Gillman MW, Pasternak LR, Peterson CL, Young WG Jr. Familial carotid body tumors: case report and epidemiologic review. Cancer 1980;46(09):2116-2122

11 Mehra S, Chung-Park M. Gallbladder paraganglioma: a case report with review of the literature. Arch Pathol Lab Med 2005;129(04): 523-526 